\title{
Construction of elastic wheels on light rail vehicles
}

\author{
Ioan Sebeșan ${ }^{1 *}$, Sorin Arsene ${ }^{1}$ and Ion Manea ${ }^{2}$ \\ ${ }^{1}$ Polytechnic University of Bucharest, Department of Railway Rolling Stock, Sector 6, Splaiul \\ Independenței, No. 313, Romania \\ ${ }^{2}$ SC Softronic SRL, R\&D Department, Craiova, No.40, Dolj, Romania
}

\begin{abstract}
In the case of urban rail transport, light vehicles can be an effective solution due to the advantages offered by the elastic wheels. One of the great advantages of these rolling elements is given by the reduction of unsuspended masses of the vehicle which have the effect of reducing the vehicle-rolling interaction forces. However, there are no other advantages to be missed, such as noise reduction (very important for urban vehicles) and improved walking quality. This paper aims at presenting the construction and design elements of the elastic wheels. It is hoped to improve the quality of the urban transport vehicles in Romania. At the same time, dynamic effects will be analysed and presented on the wheel rail contact, in the case of vehicles equipped with elastic wheels.
\end{abstract}

\section{General considerations}

The paper presents a model for calculating the dynamic loads at the wheel - rail contact. It also shows the advantages of the elastic wheels in terms of dynamic loads and vehicle sounds. Considering the frequency decoupling of the non-suspended masses vibrations to the suspended masses of the vehicle, without making too great errors, the simplified mechanical model of Figure 1 can be used to study the vibrations of the non-suspended masses.

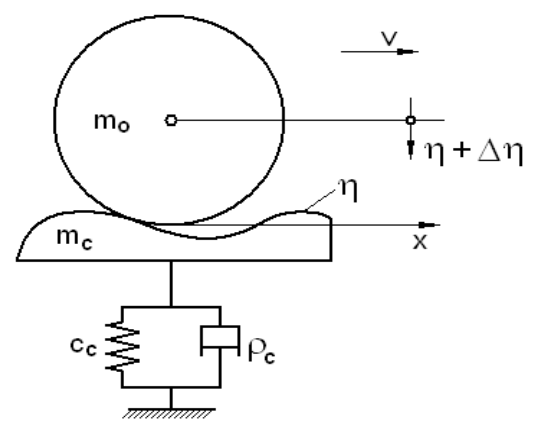

Fig. 1. Mechanical model of the unsupported mass-track system.

\footnotetext{
*Corresponding author: ioan_sebesan@yahoo.com
} 
The forced vibrations of an axle caused by a length $L$ and the maximum depth $H$, have as a mathematical model the differential equation (1):

$$
\left(m_{o}+m_{c}\right) d^{2}(\eta+\Delta \eta) / d t^{2}+c_{c} \Delta \eta=0
$$

Where: $\eta$ represents the variable depth of the grubbing under the effect of the static loads; $\Delta \eta$ - Dynamic additional flexion path; $\mathrm{c}_{\mathrm{c}}$ - rigidity of the path; $\mathrm{m}_{\mathrm{o}}$ - mass of the axle; $\mathrm{mc}$ reduced track mass (which, in relation to the axle mass, can be neglected).

From (1) the resulting pulsation results

$$
\omega_{c}=\sqrt{c_{c} /\left(m_{O}+m_{c}\right)}
$$

For example, neglecting the reduced mass of the $\mathrm{m}_{\mathrm{c}}$ path, for $m_{o}=2 \cdot 10^{3} \mathrm{~kg}$ şi $c_{c}=10^{8} \mathrm{~N} / \mathrm{m}$, is obtained $\omega_{o}=224 \mathrm{rad} / \mathrm{s}$, respectively a pulsation of its own $T_{c}=2 \pi / \omega_{c} \approx 0.028 \mathrm{~s}$ and its own frequency $v_{c}=1 / T_{c} \approx 36 \mathrm{~Hz}$, sufficiently high in relation to the own frequencies of suspended masses.

\section{Axle movement and dynamic overhangs exerted by the axle on the track}

For the study of the vertical movement of the axle, the mechanical model of Figure 1 is also considered, considering the path damping.

By denoting with $\mathrm{z}=\eta+\Delta \eta$, the axle motion equation is:

$$
m_{o} \ddot{z}+\rho_{c}(\dot{z}-\dot{\eta})+c_{c}(z-\eta)=0
$$

Or

$$
m_{o} \ddot{z}+\rho_{c} \dot{z}+c_{c} z=\rho_{c} \dot{\eta}+c_{c} \eta
$$

It is considered to be a harmonic function of the form $n=\eta_{0} \sin \omega t$, amplitude $\eta_{0}$ and pulse $\omega$.

Using complex notations

$$
\bar{\eta}=\eta_{0} e^{\mathrm{j} \omega t} ; \quad \overline{\mathrm{z}}=\mathrm{z}_{0} e^{j(\omega t+\alpha)}
$$

Where $z_{0}$ represents the amplitude of the displacement of the mass and $\alpha$ - the phase difference between the excite $\mathrm{n}$ and the displacement $\mathrm{z}$ of the axle.

With these notations, (4) the complex response factor is obtained

$$
\bar{\eta}=\eta_{0} e^{\mathrm{j} \omega t} ; \quad \overline{\mathrm{z}}=\mathrm{z}_{0} e^{j(\omega \mathrm{t}+\alpha)}
$$

Whose mode $H_{z}(\omega)=\bar{H}_{z}(\omega)$ allows to move from $\omega$ to $\mathrm{z}$.

Depending on the pulsation and the degree of damping of the path $D_{c}=\rho_{c} /\left(2 m_{c} \omega_{c}\right)=\rho_{c} /\left(2 \sqrt{c_{c} m_{o}}\right)$, is obtained

$$
H_{z}^{2}(\omega)=\frac{1+4 D_{c}^{2}\left(\omega / \omega_{c}\right)^{2}}{\left[1-\left(\omega / \omega_{c}\right)^{2}\right]^{2}+4 D_{c}^{2}\left(\omega / \omega_{c}\right)^{2}}
$$


Acceleration response factor $\ddot{z}$ will be:

$$
H_{\ddot{z}}(\omega)=\omega^{2} H_{z}(\omega)
$$

Due to the vertical vibrations of the axle, dynamic overloads marked with $\Delta \mathrm{q}$ will appear at the wheel - rail contact. The dynamic overload response factor will be

$$
H_{\Delta q}(\omega)=m_{o} H_{z}(\omega)=m_{o} \omega^{2} H_{z}(\omega)
$$

The equations $\eta(t)$ produced by the longitudinal path defects of the path having a random character, their quadratic mean is given by the relation

$$
\sigma_{n}^{2}=\frac{1}{\pi} \int_{0}^{\infty} G(\omega) \mathrm{d} \omega
$$

Where $G(\omega)$ represents the spectral power density.

From the research carried out within SNCF, which aimed to analyze the path defects and their influence on vehicles, it was concluded that $G(\omega)$ can be expressed with sufficient precision in a relationship of form

$$
G(\omega)=A v^{2} /(B v+\omega)^{2}
$$

Where A and B are constants that depend on the quality of the path.

Thus, by replacing the expression of $\mathrm{G}(\omega)$ in $(10)$, it is obtained

$$
\sigma_{\eta}^{2}=A /\left(2 \pi B^{2}\right)
$$

Which in fact represents the square of the path defects.

It has been found that for path defects with $\mathrm{L}$ wavelengths small, less than $3 \mathrm{~m}$, the term $\mathrm{B}$ in relation (11) can be neglected and therefore

$$
G(\omega)=A v^{2} / \omega^{3}
$$

In this case

$$
\sigma_{\eta}^{2}=\frac{1}{\pi} \int_{\omega_{1}}^{\infty} \frac{A v^{2}}{\omega^{3}} \mathrm{~d} \omega=\frac{A v^{2}}{2 \pi \omega_{1}^{2}}
$$

Where, $\omega_{1}=2 \pi v / L_{1}$, for $L_{1}=3 \mathrm{~m}$.

Defects with wavelengths less than $3 \mathrm{~m}$ are generally corrugated wear data. Since the frequencies of the vibrational unsustainable mass system-path are $30 \ldots 40 \mathrm{~Hz}$, short wavelength failures cause significant dynamic overloads, especially at high speeds.

The power spectral density of the vertical displacement of the axle, thus exciting the vibrant vehicle system, will be

$$
G_{z}(\omega)=H_{z}^{2}(\omega) G(\omega)=\frac{A v^{2}}{(B v+\omega)^{3}} \cdot \frac{1+4 D_{c}^{2}\left(\omega / \omega_{c}\right)^{2}}{\left[1-\left(\omega / \omega_{c}\right)^{2}\right]^{2}+4 D_{c}^{2}\left(\omega / \omega_{c}\right)^{2}}
$$

And the dynamic overload $\Delta q$. 


$$
G_{\Delta q}(\omega)=H_{\Delta q}^{2}(\omega) G(\omega)=m_{o} A v^{2} \omega \frac{1+4 D_{c}^{2}\left(\omega / \omega_{c}\right)^{2}}{\left[1-\left(\omega / \omega_{c}\right)^{2}\right]^{2}+4 D_{c}^{2}\left(\omega / \omega_{c}\right)^{2}}
$$

Average square of dynamic overloads $\Delta q$ it is

$$
\sigma_{\Delta q}^{2}=\frac{1}{\pi} \int_{0}^{\infty} G_{\Delta q}(\omega) \mathrm{d} \omega=\frac{1}{\pi} \int_{0}^{\infty} H_{\Delta q}^{2}(\omega) \cdot \mathrm{G}(\omega) \mathrm{d} \omega
$$

The smallest wavelengths are even those given by the elastic deformations of the contact surfaces. If $2 \mathrm{a}$ is the length of the contact ellipse, the highest pulsation possible is $\omega=\pi \mathrm{v} / \omega$. In view of this observation,

$$
\sigma_{\Delta q}^{2}=\frac{m_{o}^{2} A v^{2}}{\pi} \int_{0}^{\pi v / a} \frac{1+4 D_{c}^{2}\left(\omega / \omega_{c}\right)^{2}}{\left[1-\left(\omega / \omega_{c}\right)^{2}\right]^{2}+4 D_{c}^{2}\left(\omega / \omega_{c}\right)^{2}} \omega d \omega .
$$

For $m_{o}=2 \cdot 10^{3} \mathrm{~kg}, \quad \mathrm{c}_{\mathrm{c}}=10^{8} \mathrm{~N} / \mathrm{m}, \mathrm{D}_{\mathrm{c}}=0,3,2 \mathrm{a}=6 \cdot 10^{-3} \mathrm{~m}$, after completing the integration, Prud'Homme reaches the following approximate relationship:

$$
\sigma_{\Delta q}^{2}=(4 / \pi) A m_{o}^{2} \omega_{c}^{2} v^{2} \approx 1,3 A c_{c} m_{o} v^{2}
$$

From where, considering $A=2 \cdot 10^{-6}$, it is obtained

$$
\sigma_{\Delta q}^{2}=(4 / \pi) A m_{o}^{2} \omega_{c}^{2} v^{2} \approx 1,3 A c_{c} m_{o} v^{2}
$$

Which shows that dynamic overloads due to short wavelength defects vary in proportion to vehicle speed and can reach considerable values in high-speed traffic. To reduce these, it is necessary that the unassisted masses of the vehicle are as small as possible. Through their construction, the elastic wheels meet these criteria.

\section{Constructional principles and elasticity of wheels. Effects of sunburn}

The idea of breaking the metal chain that conveys shocks, vibrations and rolling noise from the wheel to the vehicle body by interposing an elastic element and closer to the exciting source has been backed up by the special advantages it offers.

The most significant reduction in the mass of the vehicle without an elastic suspension makes the disturbing influence of the path irregularities only felt on these small masses and is attenuated at the rest of the vehicle construction.

Figure 2 illustrates the substantial reduction of elastically unsustainable masses by interposing a rubber element between the rolling wheel bundle and its body in an electric locomotive whereby the traction is effected by an electromotor mounted with axle and nose bearings. On the right side of the Figure, the blackened portion represents the elastically unsustainable masses in the case of a metallic wheel. On the left side of the Figure, these masses are summed up to the bandage and about 3 to $5 \%$ of the total mass of the wheel train with a mounted motor. As a result of this reduction in unsustainable mass, the disturbing effects of the pathway are substantially reduced. 


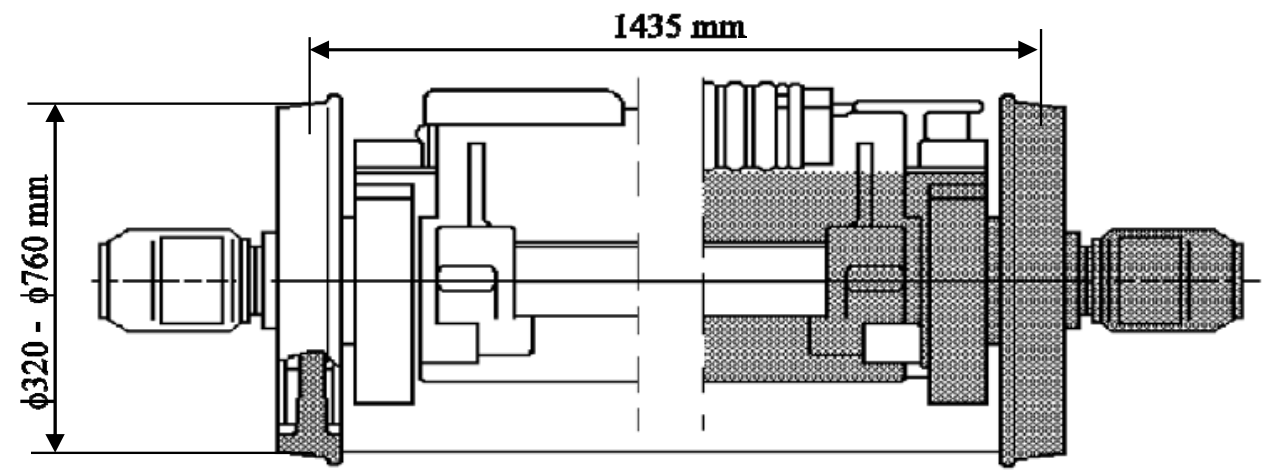

Fig. 2. Unsupported masses for metal wheels and wheels.

The effect of tread rolling elasticity is manifested by the attenuation of shocks and vibrations transmitted to the suspended mass.

Figure 3 shows the vertical accelerations az measured on the axle bearing housing in the case of standard (metallic) wheels and elastic wheels on subway cars and on railway vehicles. The higher the shock received from the tread of the wheel band, the higher the shock absorption factor transmitted to the center of the wheel and the wheel train, as well as the other vehicle organs (transmissions, motors ...).

\section{Subway in alignment}

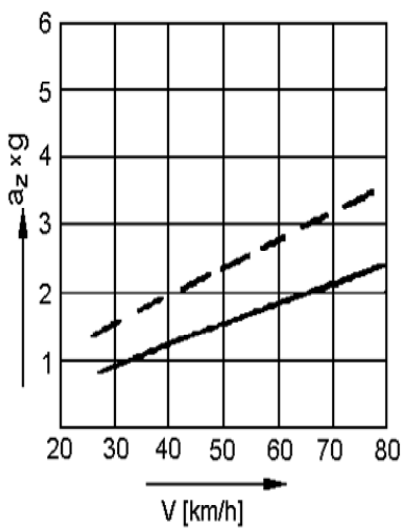

in alignment

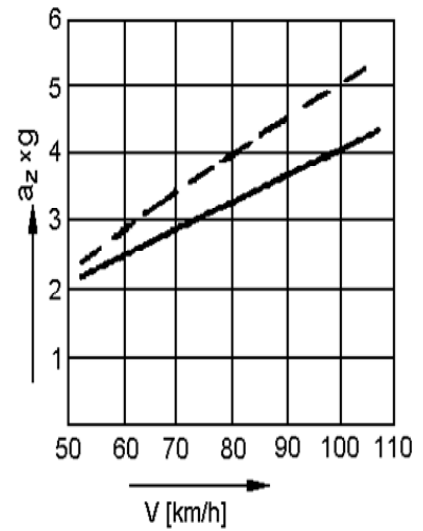

Railroad

at the switch

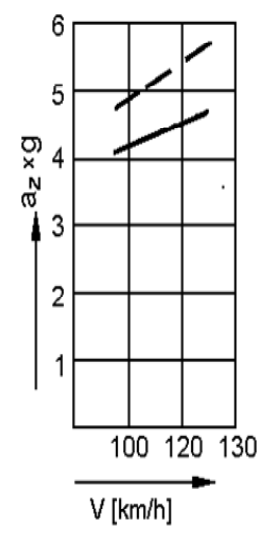

Standand wheek

Elastic wheets

Fig. 3. Elastic rolling wheel damping effect - Acceleration measured on the axle bearing (az) on elastic wheels and metal wheels.

In addition to the qualitative image resulting from Figure 3, it is possible to calculate the force demanding the axle spindle in both situations, for example, in the case of a metro at $50 \mathrm{~km} / \mathrm{h}$.

According to the diagram in Figure 3, the damping factor

$$
D=\frac{a_{z \text { elastic }}}{\mathrm{a}_{\mathrm{z} \text { rigid }}}=\frac{1,5 g}{2,2 g}=0,68,
$$

Respectively, the dynamic force transmitted to the axle shaft is $32 \%$ lower in the case of the elastic wheel. In general, an average damping factor of $25 \ldots 30 \%$ for vertical accelerations (z) can be relied upon. 
Wheel elasticity also greatly contributes to reducing rolling noise, both inside and outside the vehicles, due to the fact that rolling vibrations are transmitted attenuated to the wagon organs which generally amplify these vibrations (the tympanum formed by the center wheel, vehicle body, etc.)

\section{Conclusions}

Elastic wheels are used in urban vehicles with low axle loads, especially in subway vehicles. They have to meet the two basic requirements specific to railway vehicles, namely to ensure the safety of the guiding and the quality of the ride, especially since such vehicles fall under the "hard" mode of transport.

From the presented in the paper it is shown the necessity of reducing the unsustainable masses of the vehicle. This reduces the dynamic wheel-to-rail forces, thus improving the safety of the guiding, but also the overall driving quality of the vehicle.

The elastic wheels as unsustainable mass can be considered the mass of the crown of the wheel by running on the rails in direct contact. The rubber elements of the wheel assure the elastic uptake of the interaction forces in the vertical, transverse and longitudinal directions. The magnitude of accelerations measured on the axles shows an important reduction in the elastic wheels relative to the measured accelerations for conventional metal wheels.

At the body of the vehicle, the elastic wheels provide a low-vibration vibration regime. Taking into account the effect of resonance of the elastic wheels, it is concluded that a vehicle with such wheels achieves a good quality of walking.

This work was supported by a grant of the Romanian National Authority for Scientific Research and Innovation, CNCS-UEFISCDI, project number PN-III-P2-2.1-PTE-2016 -0008, within PNCDI III

\section{References}

1 I. Sebeșan, C. Crăciun, M. St. Sebeșan Analysis and calculation models of shock and vibration isolation systems on railway vehicles, MatrixRom, Bucharest, (2016)

2 I. Sebeșan, D. Băiașu Mechanical patterns in the dynamics of railway vehicles, Ed. Romanian Academy, Bucharest, (2014)

3 C. Anderson, J. Oscarson, Veh. Syst. Dyn., 33, (1999)

4 PN - II - PT - PCCA - 2013 - 3, no.192/2012, Solutions to Improve Dynamic Performance and Impact Safety of Railway Traction Vehicles to Align to Requirements Required by European Standards (2012)

5 I. Sebesan, M. A. Spiroiu, S. Arsene, G. Popa, G. Dinu, Mat. Plast., 52, 4, 560-563 (2015) 